\title{
Binary Neutron Stars in General Relativity: Quasiequilibrium Models
}

\author{
T. W. Baumgarte, ${ }^{1}$ G. B. Cook, ${ }^{2}$ M. A. Scheel, ${ }^{2}$ S. L. Shapiro, ${ }^{1,3}$ and S. A. Teukolsky ${ }^{2,4}$ \\ ${ }^{1}$ Department of Physics, University of Illinois at Urbana-Champaign, Urbana, Illinois 61801 \\ ${ }^{2}$ Center for Radiophysics and Space Research, Cornell University, Ithaca, New York 14853 \\ ${ }^{3}$ Department of Astronomy and NCSA, University of Illinois at Urbana-Champaign, Urbana, Illinois 61801 \\ ${ }^{4}$ Departments of Physics and Astronomy, Cornell University, Ithaca, New York 14853
}

(Received 8 April 1997)

\begin{abstract}
We perform fully relativistic calculations of binary neutron stars in quasiequilibrium circular orbits. We integrate Einstein's equations together with the relativistic equation of hydrostatic equilibrium to solve the initial-value problem for equal-mass binaries of arbitrary separation. We construct sequences of constant rest mass and identify the innermost stable circular orbit and its angular velocity. We find that the quasiequilibrium maximum allowed mass of a neutron star in a close binary is slightly larger than in isolation. [S0031-9007(97)03845-3]
\end{abstract}

PACS numbers: 04.25.Dm, 04.30.Db, 04.40.Dg, 97.60.Jd

The two-body problem is one of the outstanding, unsolved problems in classical general relativity. And yet, neutron star binary systems are known to exist, even within our own galaxy [1]. For some of these systems (including PSR B1913+16 and B1534+12) general relativistic orbital effects have been measured to high precision [2]. Binary neutron stars are among the most promising sources for gravitational wave detectors now under construction, like LIGO, VIRGO, and GEO. This has triggered an intense theoretical effort to predict the gravitational wave form emitted during the inspiral and coalescence of the two stars.

Much of the work on binary neutron stars has been performed within the framework of Newtonian hydrodynamics [3]. Many investigators have also studied the problem in post-Newtonian (PN) theory. As long as the PN stars are well separated, they can be approximated by point sources [4], but for close binaries, hydrodynamical effects must also be taken into account [5-9].

Fully general relativistic treatments of the problem are complicated by the nonlinearity of Einstein's equations and the requirement of very large computational resources. Numerical simulations are currently only in their infancy [6]. Recently, Wilson and Mathews [10] reported preliminary results obtained with a relativistic numerical evolution code. Their dynamical calculations suggest that the neutron stars may collapse to black holes prior to merger. They also find that, typically, binaries have too large a total angular momentum to form a Kerr black hole immediately upon merger (see also [11]). Their results are in disagreement with predictions of Newtonian [12] and PN calculations [7], which show that tidal fields stabilize neutron stars against radial collapse.

In this Letter we report the first calculations in full relativity of quasiequilibrium, equal mass, neutron star binaries in synchronized circular orbits. We numerically integrate a subset of the Einstein equations, coupled to the equations of relativistic hydrodynamics, to solve the initial value problem for binaries. Such quasiequilibrium models provide initial data for future dynamical evolution calculations. We construct quasiequilibrium sequences of constant rest mass configurations at varying separation. These sequences mimic evolutionary sequences in which the stars undergo slow inspiral on nearly circular orbits due to the emission of gravitational waves. We identify the innermost stable circular orbit (ISCO), its angular velocity, and the maximum quasiequilibrium mass of a neutron star in a close binary.

In Newtonian gravity, strict equilibrium for two stars in synchronized circular orbit exists. Since this solution is stationary, the hydrodynamical equations reduce to the Bernoulli equation, which greatly simplifies the problem. Because of the emission of gravitational waves, a binary in general relativity cannot be in strict equilibrium. However, outside the ISCO, the timescale for orbital decay by radiation is much longer than the orbital period, so that the binary can be considered to be in "quasiequilibrium". This fact allows us to neglect both gravitational waves and wave-induced deviations from a circular orbit to very good approximation. Some of our approximations have been used and calibrated elsewhere $[13,14]$, and a more detailed discussion will be presented in a forthcoming paper [15]. Here we will briefly outline our method and present some of our key results.

We attempt to minimize the gravitational wave content in the solution, in compliance with physical expectations, by choosing the 3 -metric to be conformally flat $[10,13]$. In Cartesian coordinates the line element can then be written

$$
d s^{2}=-\alpha^{2} d t^{2}+\Psi^{4} \delta_{i j}\left(d x^{i}-\omega^{i} d t\right)\left(d x^{j}-\omega^{j} d t\right)
$$

where $\alpha$ is the lapse, $\omega^{i}$ the shift, and $\Psi$ the conformal factor. We satisfy the initial value equations of relativity precisely. Our approximation lies in assuming that the metric will remain conformally flat for all times. The 
extrinsic curvature $K_{i j}$ then has to satisfy

$$
\bar{K}^{i j}=-\frac{\Psi^{6}}{2 \alpha}\left(\nabla^{i} \omega^{j}+\nabla^{j} \omega^{i}-\frac{2}{3} \delta^{i j} \nabla_{k} \omega^{k}\right),
$$

where $\bar{K}^{i j}=\Psi^{10} K^{i j}$ and where we have also used the maximal slicing condition $K_{i}^{i}=0$. Here $\nabla^{i}$ is the flat space derivative operator in Cartesian coordinates.

We assume that the matter obeys a polytropic equation of state

$$
P=K \rho_{0}^{1+1 / n},
$$

where $P$ is the pressure, $\rho_{0}$ the rest-mass density, $K$ the polytropic constant, and $n$ the polytropic index. We assume that we can neglect deviations from a strictly periodic circular orbit and that the stars are corotating, which is equivalent to assuming that the fluid fourvelocity is proportional to a Killing vector. In this case the matter equations can be integrated analytically, which yields the relativistic Bernoulli equation

$$
q=\frac{1}{1+n}\left(\frac{1+C}{\alpha\left(1-v^{2}\right)^{1 / 2}}-1\right),
$$

where $q=P / \rho_{0}, C$ is a constant of integration, and $v$ is the proper velocity of the matter.

The Hamiltonian constraint can now be written

$$
\begin{aligned}
\nabla^{2} \Psi= & -\frac{1}{8} \Psi^{-7} \bar{K}_{i j} \bar{K}^{i j} \\
& -2 \pi \Psi^{5} q^{n}\left(\frac{1+(1+n) q}{1-v^{2}}-q\right) .
\end{aligned}
$$

Requiring that the maximal slicing condition be maintained at all times, we can use the time evolution equation for $K_{i j}$ to find an equation for the lapse,

$$
\begin{aligned}
\nabla^{2} \tilde{\alpha}= & \frac{7}{8} \tilde{\alpha} \Psi^{-8} \bar{K}_{i j} \bar{K}^{i j} \\
& +2 \pi \tilde{\alpha} \Psi^{4} q^{n}\left((1+(n+1) q) \frac{1+2 v^{2}}{1-v^{2}}+5 q\right),
\end{aligned}
$$

where $\tilde{\alpha}=\Psi \alpha$. The momentum constraint becomes

$$
\begin{aligned}
\nabla^{2} \omega^{i}+\frac{1}{3} \nabla^{i}\left(\nabla_{j} \omega^{j}\right)= & -2 \nabla_{j}\left(\tilde{\alpha} \Psi^{-7}\right) \bar{K}^{i j} \\
& -16 \pi \Psi^{4} q^{n} \frac{1+(1+n) q}{1-v^{2}} \\
& \times\left(\Omega \xi^{i}-\omega^{i}\right),
\end{aligned}
$$

where $\Omega$ is the constant angular velocity and $\xi^{i}$ is a three-vector tangent to the matter velocity. With the stars centered along the $z$ axis and orbiting around the $y$ axis, we have $\xi^{i}=(z, 0,-x)$. The last equation can be simplified by writing $\omega^{i}=G^{i}-\frac{1}{4} \nabla^{i} B$.

Our approximations reduce the Einstein field equations to a set of coupled, quasilinear elliptic equations for the lapse, shift, and the conformal factor [Eqs. (5)-(7)] which have to be solved together with the matter equation
(4). For boundary conditions at large radius we impose asymptotic flatness. Solving these equations yields a valid solution to the initial value (constraint) equations. Such a solution will also provide an approximate instantaneous snapshot of a binary evolved according to the full Einstein equations, prior to plunge. In the Newtonian limit, the above equations reduce to the coupled Poisson and Bernoulli equations.

Our numerical implementation will be described in detail in [15]. Since the stars have equal mass, it is sufficient to work in one octant only. We use a full approximation storage multigrid scheme to solve the elliptic field Eqs. (5) -(7) for a given matter distribution. Once a solution has been found, the matter can be updated [Eq. (4)]. This iteration can be repeated until convergence is achieved to a desired accuracy. We have implemented this algorithm in a parallel environment using DAGH software [16]. Typical runs were computed on a grid of $64^{3}$ gridpoints. We adjusted the outer boundaries for each separation so that the matter was always covered by 17 gridpoints along the diameter.

We determine the rest (baryon) mass $M_{0}$, the total (ADM) mass-energy $M$, and the angular momentum $J$, which refer to the parameters of one individual star. Physical dimensions enter the problem only through the polytropic constant $K$ in (3). It is therefore convenient to introduce the dimensionless quantities $\bar{\rho}_{0}=K^{n} \rho_{0}, \bar{M}_{0}=$ $K^{-n / 2} M_{0}$, and $\bar{M}=K^{-n / 2} M$ (see [17]).

In the following we will discuss results for $n=1$, for which the maximum mass of a neutron star in isolation is $\bar{M}_{0}^{\max }=0.180$. In Fig. 1 we show density profiles for highly relativistic neutron stars of rest mass $\bar{M}_{0}=0.178$

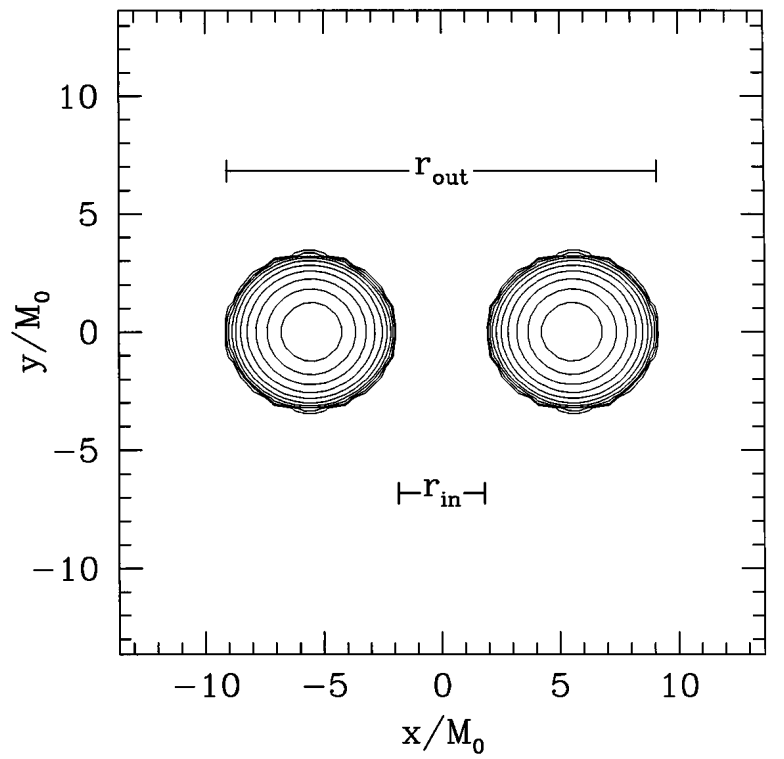

FIG. 1. Rest-density contours in the equatorial plane for a neutron star binary close to the ISCO. Each star has a rest mass of $\bar{M}_{0}=0.178$, only slightly below the maximum mass at infinite separation, $\bar{M}_{0}^{\max }=0.180$. The contours span densities between the central density and $1 \%$ of that value by decreasing factors of 0.63 . 
close to the ISCO. We define the ratio between the inner and outer coordinate separation, $z_{A}=r_{\text {in }} / r_{\text {out }}$.

In Fig. 2 we plot the allowed rest mass versus the central density for several different separations between $z_{A}=$ 0.3 (roughly two stellar radii apart) to $z_{A}=0$ (touching). As $z_{A} \rightarrow 1$ we expect these curves to approach the spherical Oppenheimer-Volkoff (OV) result, which we included as the dashed line in Fig. 2 [18]. All our graphs lie within $2 \%$ of the OV curve, showing that the presence of a companion star has only very little influence on the mass-density equilibrium relationship.

As we decrease the separation, the mass supported by a given $\bar{\rho}_{\mathrm{c}}$ increases slightly. In particular, the maximum mass increases from $\bar{M}_{0}^{\max }=0.179$ for $z_{A}=$ 0.3 to $\bar{M}_{0}^{\max }=0.182$ for touching stars. This trend clearly suggests that the maximum allowed mass of neutron stars in close binaries is slightly larger than in isolation. This increase is caused partly by the rotation of the stars and partly by the tidal fields [19]. Note, however, that we are only constructing quasiequilibrium configurations, which may or may not be dynamically stable. For nonrotating, isolated stars the maximum mass configuration in Fig. 2 marks the onset of radial instability. No general theorem can be trivially applied to binary stars. Figure 2 also shows that keeping the rest mass fixed, the central density slightly decreases as the stars approach each other and become tidally deformed. Both effects are consistent with simple PN predictions [7,9].

The collapse of binary neutron stars to black holes prior to merger reported in [10] could, in principle, be caused either by a decrease of the maximum allowed mass, or by a dynamical instability. Our results, within our approximations, rule out the first possibility.

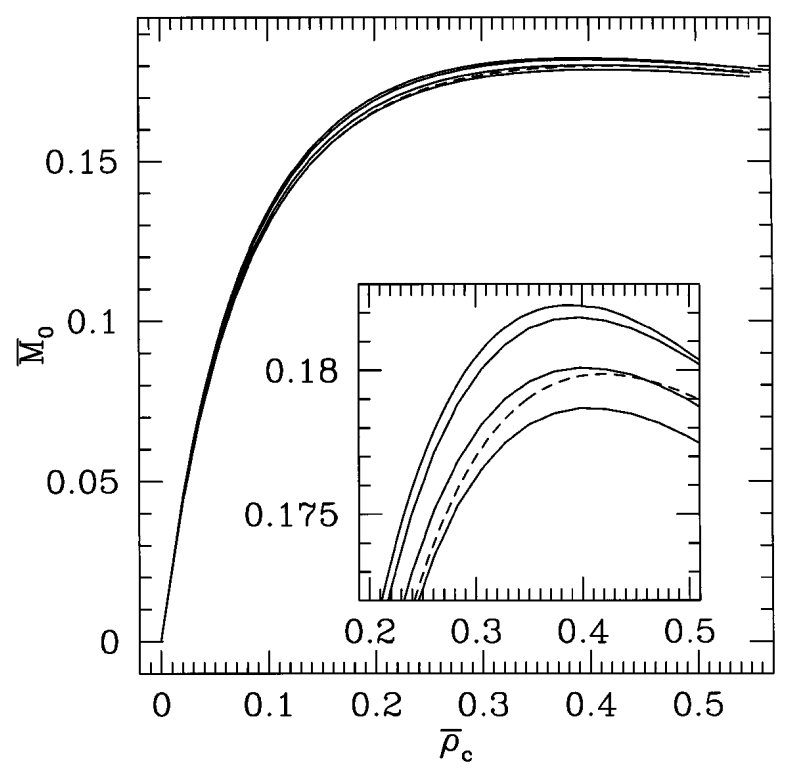

FIG. 2. Rest mass $\bar{M}_{0}$ versus central density $\bar{\rho}_{\mathrm{c}}$ for separations $z_{A}=0.3$ (bottom solid line), 0.2, 0.1 , and 0.0 (top line). The dashed line is the Oppenheimer-Volkoff result. The inset is a blowup of the region around the maximum masses.
We construct sequences of constant rest mass $\bar{M}_{0}$, which approximate evolutionary sequences up to the ISCO [20]. Note that we have assumed the stars to be corotating. This may not be realistic, since it would require excessive viscosity [21]. It is more likely that circulation of the stars is conserved during inspiral, and the stars remain nearly irrotational. Nevertheless, we expect that our sequences are a reasonable approximation to the inspiral up to the ISCO and correctly reveal the effects of nonlinear gravitation.

In Fig. 3 we show the binary binding energy versus angular velocity for several different rest masses $\bar{M}_{0}$. As the stars approach, both finite size effects and nonlinear gravitation play an increasingly important role and cause, for stiff enough equations of state, the binding energy to go through a minimum and increase again. The location of the minimum marks the onset of a secular instability, beyond which the binary can no longer maintain circular equilibrium. It is expected that the dynamical instability, which defines the true ISCO for plunge, occurs beyond, but close to, the onset of the secular instability [12]. Figure 3 shows that the orbital frequency at the ISCO $f_{\text {ISCO }}$ strongly depends on $(M / R)_{\infty}$. For small $(M / R)_{\infty}$ we find small values of $f_{\text {ISCO }}$ very similar to those reported in [10], while for larger $(M / R)_{\infty} f_{\text {ISCO }}$ increases to frequencies that will be detectable by gravitational wave detectors currently under construction.

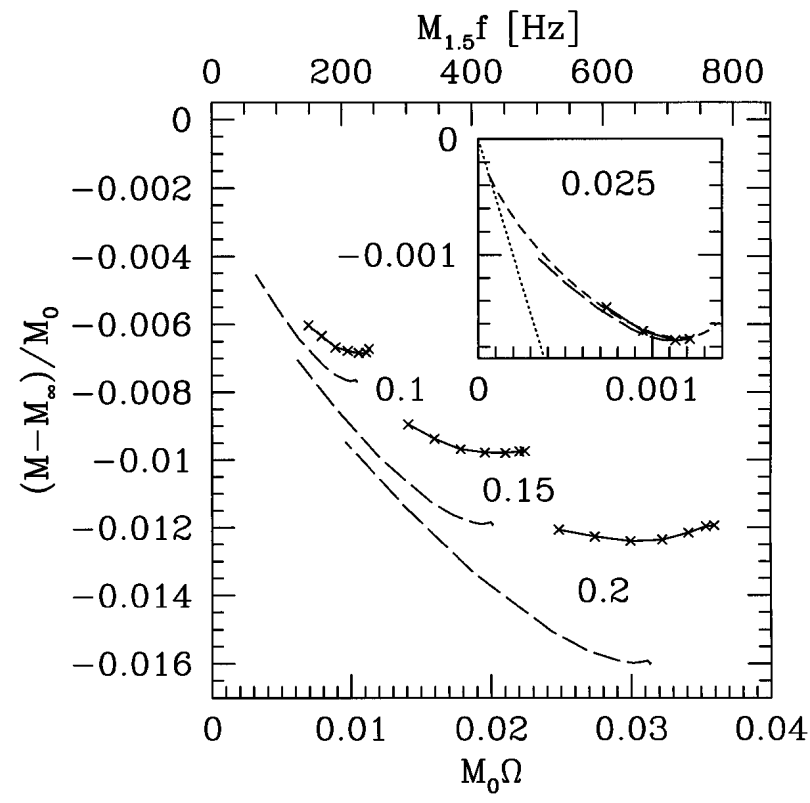

FIG. 3. The binding energy as a function of the angular velocity for different rest masses $\bar{M}_{0}$. The curves are labeled by the compaction $(M / R)_{\infty}$ of the stars in isolation at infinity. The maximum compaction for a stable, isolated, nonrotating $n=1$ polytrope is 0.217 . The upper label gives the orbital frequency for stars with a rest mass of $1.5 M_{\odot}$. Dashed curves are the corresponding results from a Newtonian version of our code. In the inset we plot results for a nearly Newtonian configuration with $(M / R)_{\infty}=0.025$. Here we have also included the results from a Newtonian ellipsoidal treatment [12] (long dashed line) and for two point particles (dotted line). 
TABLE I. Numerical values for sequences of constant rest mass $\bar{M}_{0}$ and polytropic index $n=1$. We tabulate the total energy $\bar{M}_{\infty}$ and compaction $(M / R)_{\infty}$ each star would have in isolation at infinity as well as the angular velocity $M_{0} \Omega$ and the angular momentum $J_{\text {tot }} / M_{\text {tot }}^{2}$ at the ISCO.

\begin{tabular}{ccccc}
\hline \hline $\bar{M}_{0}$ & $\bar{M}_{\infty}$ & $(M / R)_{\infty}$ & $M_{0} \Omega_{\text {ISCO }}$ & $\left(J_{\text {tot }} / M_{\text {tot }}^{2}\right)_{\text {ISCO }}$ \\
\hline 0.112 & 0.106 & 0.1 & 0.01 & 1.22 \\
0.134 & 0.126 & 0.125 & 0.015 & 1.12 \\
0.153 & 0.142 & 0.15 & 0.02 & 1.05 \\
0.169 & 0.155 & 0.175 & 0.025 & 1.00 \\
0.178 & 0.162 & 0.2 & 0.03 & 0.97 \\
\hline \hline
\end{tabular}

In the Newtonian regime our results agree very well with both a Newtonian version of our code and results from an ellipsoidal treatment of the binaries [12] (see inset). For more relativistic models, comparisons are made somewhat ambiguous by the adopted choice of a parameter to characterize the sequence. Identifying the member at infinity by its value of $M$ (or $M_{0}$ ) versus $(M / R)_{\infty}$ (or $\left.\left(M_{0} / R\right)_{\infty}\right)$ leads to different Newtonian models and binding energy curves. In Fig. 3 we choose $\left(M_{0} / R\right)_{\infty}$ and find that the ISCO frequencies agree closely with the Newtonian values, but the binding energies differ as the compaction increases (see also [5,8,9]).

We summarize these results in Table I, where we also tabulate the dimensionless total angular momentum $J_{\text {tot }} / M_{\text {tot }}^{2}=J / 2 M^{2}$ at the ISCO. For small rest masses this value is larger than unity, in agreement with [10]. For high enough rest masses, however, it drops below unity, so that the two stars could plunge and form a Kerr black hole without having to radiate additional angular momentum. Because the orbit will decay rapidly inside the ISCO, its presence will leave a measurable imprint on the emitted gravitational wave form. Measuring $\Omega_{\mathrm{ISCO}}$ may be the crucial ingredient in determining the radius of the star, assuming that the mass has been determined during the prior inspiral phase [22].

It is a pleasure to thank Manish Parashar for his help with the implementation of DAGH, and Andrew Abrahams, James Lombardi, and Fred Rasio for several helpful discussions. This work was supported by NSF Grant AST 96-18524 and NASA Grant NAG 5-3420 at Illinois, NSF Grant PHY 94-08378 at Cornell, and by the NSF Binary Black Hole Grand Challenge Grant Nos. NSF PHY 93-18152/ASC 93-18152 (ARPA supplemented).

[1] S.E. Thorsett, Z. Arzoumanian, M. M. McKinnon, and J. H. Taylor, Astrophys. J. 405, L29 (1993).

[2] J.H. Taylor and J. M. Weisberg, Astrophys. J. 345, 434 (1989); Z. Arzoumanian, Ph.D. Thesis, Princeton University (1995).

[3] See, for example, I. Hachisu and Y. Eriguchi, Publ. Astron. Soc. Jpn. 36, 239 (1984); M. Shibata, T. Nakamura, and K. Oohara, Prog. Theor. Phys. 88, 1079 (1992); F. A.
Rasio and S. L. Shapiro, Astrophys. J. 432, 242 (1994); X. Zhuge, J. M. Centrella, and S. L. W. McMillan, Phys. Rev. D 54, 7261 (1996); M. Ruffert, H.-T. Janka, and G. Schäfer, Astrophys. Sp. Sci. 231, 423 (1995).

[4] L. Blanchet, T. Damour, B. R. Iyer, C. M. Will, and A. G. Wiseman, Phys. Rev. Lett. 74, 3515 (1995).

[5] M. Shibata, Prog. Theor. Phys. 96, 317 (1996).

[6] K. Oohara and T. Nakamura, Lecture delivered at Les Houches School "Astrophysical Sources of Gravitational Radiation" (Les Houches, France, Sept. 26-Oct. 6, 1995). To be published in the Proceedings (edited by J.-A. Marck and J.-P. Lasota) (see also Report No. astro-ph/9606179).

[7] D. Lai, Phys. Rev. Lett. 76, 4878 (1996).

[8] D. Lai and A. G. Wiseman, Phys. Rev. D 54, 3958 (1996).

[9] J.C. Lombardi, F. A. Rasio, and S. L. Shapiro, 1997, submitted (see also Report No. astro-ph/9705218).

[10] J. R. Wilson and G. J. Mathews, Phys. Rev. Lett. 75, 4161 (1995); J. R. Wilson, G. J. Mathews, and P. Marronetti, Phys. Rev. D 54, 1317 (1996).

[11] D. M. Eardley and E. W. Hirschmann, Report No. NSF-ITP-95-165 (1995) (see also Report No. astro-ph/ 9601019).

[12] D. Lai, F. A. Rasio, and S. L. Shapiro, Astrophys. J. Suppl. 88, 205 (1993).

[13] J. R. Wilson and G. J. Mathews, in Frontiers in Numerical Relativity, edited by C.R. Evans, L.S. Finn, and D.W. Hobill (Cambridge University Press, Cambridge, England, 1989), p. 306.

[14] G. B. Cook, S. L. Shapiro, and S.A. Teukolsky, Phys. Rev. D 53, 5533 (1996).

[15] T. W. Baumgarte, G. B. Cook, M. A. Scheel, S. L. Shapiro, and S. A. Teukolsky (to be published).

[16] M. Parashar and J.C. Brown, in Proceedings of the International Conference for High Performance Computing, edited by S. Sahni, V. K. Prasanna, and V. P. Bhatkar (Tata McGraw-Hill Publishing Company Ltd, 1995); see also www.ticam.utexas.edu/ parashar/public_html/ DAGH/.

[17] G. B. Cook, S. L. Shapiro, and S. A. Teukolsky, Astrophys. J. 422, 227 (1994).

[18] Computing the Oppenheimer-Volkoff curve with a very high radial resolution yields a maximum mass which is slightly larger than the masses of some of our binary models. However, we know from convergence tests that we underestimate the masses of the binary configurations by a few percent as a result of our coarse numerical grid.

[19] We find an increase in the maximum allowed mass that is comparable to the increase of the maximum mass of an isolated neutron star rotating with the same angular velocity (see [17]). Any destabilizing, relativistic effect in binaries therefore has to be smaller.

[20] As a code test we checked that the law $d M=\Omega d J$ is well satisfied along these sequences [see J. B. Hartle, Astrophys. J. 161, 111 (1970)].

[21] L. Bildsten and C. Cutler, Astrophys. J. 400, 175 (1992); C. S. Kochanek, Astrophys. J. 398, 234 (1992).

[22] K. S. Thorne, in Proceedings of the Snowmass Summer Study on Particle and Nuclear Astrophysics and Cosmology, Snowmass, Colorado, 1994, edited by E. W. Kolb and R. Pecci (World Scientific, Singapore, 1995). 